\title{
Top Physics at the LHC
}

\section{Dominique Pallin}

Laboratoire de Physique Corpusculaire

Université Blaise Pascal IN2P3/CNRS

24, Av. des Landais, Aubière, France

E-mail: pallin@clermont.in2p3.fr

\begin{abstract}
In less than two years from now, the Large Hadron Collider (LHC) will provide first proton-proton collisions at $\sqrt{s}_{\mathrm{s}}=14 \mathrm{TeV}$. The LHC will be a "Top factory" since about 8 millions of top-antitop events will be produced in one year at low luminosity. After the Top quark discovery and first measurements at Tevatron, the LHC will open a new opportunity for precision measurements of the Top quark properties. Prior to data taking, ATLAS and CMS detectors have to be commissioned and precisely calibrated. Further improvements will be achieved with first collisions. Due to the large amount of produced events and clean signal, early Top signals will play an important role in commissioning the detectors. Moreover some of the early LHC physics results could come from Top physics, leading to a major improvement of Top quark understanding and eventually opening a window for physics beyond the standard model.
\end{abstract}

International Workshop on Top Quark Physics

University of Coimbra, Portugal

12-15 January, 2006

\section{${ }^{*}$ Speaker}




\section{LHC: A new Factory for Top quarks}

Since its discovery in 1995 at Tevatron, the Top quark is studied with an increasing level of precision according to the amount of data collected by CDF and DO experiments. However most of the measurements are still statistically limited and larger Top samples are needed to reveal the true nature of the Top quark and to open a window on new physics.

Data taking at Tevatron will continue up to the end of the decade allowing CDF and D0 experiments to perform precision measurements on the Top. In the meantime, a new collider, the Large Hadron Collider will enter into operation with a great potential for precision measurements of Top quark properties. This report gives an overview of the status and plans for the machine and detectors, gives some examples of Top studies during the detectors commissioning and summarizes the LHC potential for Top quark physics from early data to precision measurements with $10 \mathrm{fb}^{-1}$ of collected data.

\subsection{The Large Hadron Collider, the ATLAS and CMS experiments}

The Large Hadron Collider, LHC, is in construction in the 27km LEP ring at CERN. 1232 superconducting dipole magnets with $8.33 \mathrm{~T}$ magnetic field will provide proton-proton collisions at a center-of-mass energy of $14 \mathrm{TeV}$, seven times larger than the current highest energy achieved at the Tevatron. At the design luminosity of $10^{34} \mathrm{~cm}^{-2} \mathrm{~s}^{-1}, 2808$ bunches per beam with a nominal intensity of $1.110^{11}$ protons/bunch and a bunch spacing of $25 \mathrm{~ns}$ will circulate in the rings.

The machine construction is performing well and should be completed soon. The LHC schedule is monthly updated and can be tracked through the LHC dashboard page available from the CERN homepage [1] which indicates that the LHC will be completed by the end of 2006, with the machine commissioning starting in April 2007. The start-up of the machine is planned in four stages, with a continuous ramping to the design machine parameters. The current schedule is summarized here:

- First collisions are expected in summer 2007, the luminosity during the pilot run in fall 2007 being of the order of 1 hundredth of the design luminosity.

- In 2008, a physics run of several months at $10^{33} \mathrm{~cm}^{-2} \mathrm{~s}^{-1}$.

- Then a ramping up to the design luminosity which could be reached by the end of 2010.

Even if it is expected to collect $10 \mathrm{fb}^{-1}$ per year during the low luminosity phase $\left(10^{33} \mathrm{~cm}^{-}\right.$ ${ }^{2} \mathrm{~s}^{-1}$ ) and up to $100 \mathrm{fb}^{-1}$ per year at design luminosity, large uncertainties exist on this schedule since it is difficult to predict how fast will be the ramping up toward the nominal luminosity. It is assumed [4] that the integrated luminosity collected by ATLAS and CMS would be in the range $0.1-10 \mathrm{fb}^{-1}$ by the end of 2008 .

Four interaction regions have been constructed. Two of them are hosting general purpose experiments ATLAS [2] and CMS [3], designed for studying with high precision pp collision at high luminosity. The two detectors are currently under installation. 


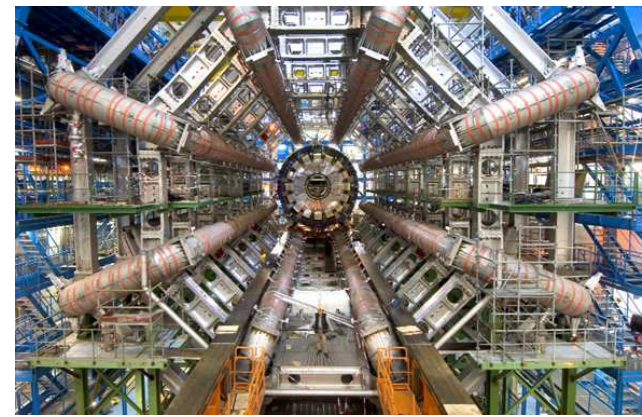

Figure 1: View through the ATLAS Barrel Toroid, just before the barrel calorimeter is moved in. [5]

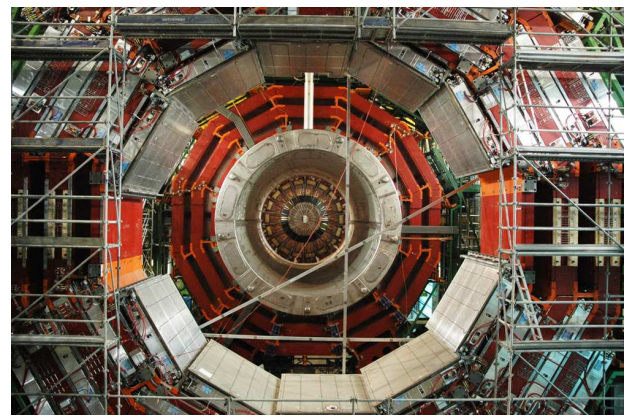

Figure 2: View through CMS during the cool down of the solenoid. [6]

The progress in the installation of the ATLAS detector is clearly visible on Figure 1. The eight barrel toroid coils of the muon system are now installed and fill up the experimental cavern. The barrel part of the calorimeters with the cryostat holding the electromagnetic calorimeter and the superconducting solenoid have been put in final position inside the toroids. The hadronic tile calorimeter has already registered first cosmic rays in June 2005. Currently, the first end-cap part of the calorimeters is completed in the pit.

CMS assembly takes place on the surface (Figure 2). The magnetic return yoke is instrumented with muons chambers and the large solenoid insertion is completed. The first cooldown of the solenoid started and will be followed by the magnet test challenge in spring 2006. Then the five barrel rings and the two endcaps will be lowered in huge segments into the underground experimental cavern to form the CMS detector.

Table 1 : Examples of expected detector performance for ATLAS and CMS at the time of the LHC start-up, and physics samples which will be used to improve this performance (from [4])

\begin{tabular}{|l|c|c|}
\hline & $\begin{array}{c}\text { Expected performance } \\
\text { on "day 1" }\end{array}$ & $\begin{array}{c}\text { Data samples (examples) to } \\
\text { improve the performance }\end{array}$ \\
\hline ECAL uniformity & $\sim 1 \%(\sim 4 \%)$ in ATLAS (CMS) & Minimum bias, $\mathrm{Z} \rightarrow \mathrm{ee}$ \\
\hline Electron energy scale & $1-2 \%$ & $\mathrm{Z} \rightarrow \mathrm{ee}$ \\
\hline HCAL uniformity & $2-3 \%$ & Single pions, QCD jets \\
\hline Jet energy scale & $\leq 10 \%$ & $\mathrm{Z}(\rightarrow \mathrm{ll})+$ jet, $\mathrm{W} \rightarrow \mathrm{jjin} \overline{\mathrm{tt}}$ events \\
\hline Tracker alignment & $20-200 \mu \mathrm{m}$ in $\mathrm{R} \phi$ & Generic tracks, isolated $\mu, \mathrm{Z} \rightarrow \mu \mu$ \\
\hline
\end{tabular}

Overall installations will be finished by June 2007. Nevertheless both detectors will not be complete. The electronic calorimeter of CMS End-caps and the CMS pixel detector will be installed during the first shutdown after the pilot run in 2007. ATLAS will start with a Transition Radiation Tracker coverage reduced to $|\eta|<2$ instead of $|\eta|<2.4$. Both experiments will have a reduced trigger bandwidth due to deferrals on HLT processors. 
In addition to test beam measurements and simulation studies, cosmic muons and then beam-halo muons or beam-gas events produced when one beam is circulating in the machine will be the basic ingredients to understand the detector performance, initial alignment and to improve calibration before commissioning with first real collisions [7]. Table 1 gives some indications on the expected initial detector performance at the time of first collisions in LHC [4]. Various physics samples will then be used to improve the performance with first set of collected pp collisions. For example, $\mathrm{Z}(\rightarrow \mathrm{ll})+$ jet and $\mathrm{W} \rightarrow \mathrm{jj}$ in $\overline{\mathrm{t} t}$ events will be used to reach quickly a few per-cent uncertainty on the absolute jet scale.

\section{Top physics}

\subsection{Top Production and decay at LHC}

With the increase of the center-of-masse energy to $14 \mathrm{TeV}$, cross-sections are higher at LHC than at Tevatron as illustrated in Table 2. For example the rate of the dominant process for Top production is about 100 times larger than at $\sqrt{\mathrm{s}}=1.96 \mathrm{TeV}$, leading to a factor thousand in Top event production at LHC for one year data taking at $10^{33} \mathrm{~cm}^{-2} \mathrm{~s}^{-1}$ with respect to Tevatron. Within one year, LHC experiments will record a larger amount of Top events than ever accumulated at previous colliders. This makes the LHC a Top factory thus opening a great opportunity to perform precision measurements in the Top quark sector.

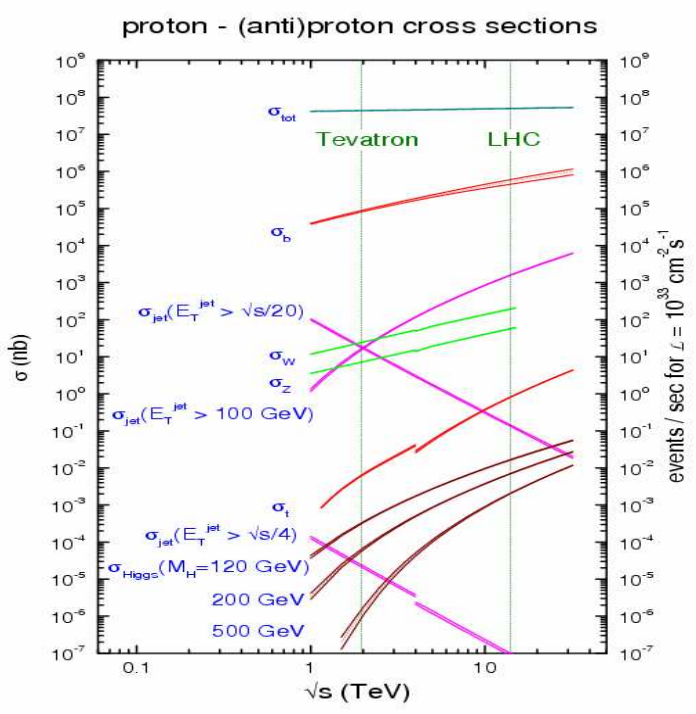

Table $2: \mathrm{pp}$ and $\mathrm{p} \overline{\mathrm{p}}$ production cross sections for various processes as function of the centre-ofmass energy. 
Top quarks are mainly produced as unpolarised $\overline{\mathrm{t}}$ pairs via pair production mechanisms. At LHC energies the hard process $\mathrm{gg} \rightarrow \overline{\mathrm{t}}$ contributes to $90 \%$ of the total $\overline{\mathrm{t}}$ cross section (the quark annihilation process accounts for the remaining 10\%) according to the large gluon component in the proton parton distributions. The most recent cross-section prediction at nextto-next-to-leading order (NNLO) including soft-gluon corrections is $\sigma(\overline{t t})=873 \mathrm{pb}$ for $\mathrm{m}_{\mathrm{t}}=175 \mathrm{GeV}$ [8]. Thus, more than 8 millions of $\overline{\mathrm{tt}}$ events are expected to be produced per year at low luminosity $\left(10 \mathrm{fb}^{-1}\right)$. In the SM the Top quark decays before hadronisation and almost exclusively into a $\mathrm{W}$ boson and a $\mathrm{b}$ quark $\mathrm{t} \rightarrow \mathrm{Wb}$. The signature of the Top-pair final states 'di-leptons', 'lepton+jets' and 'full hadronic' - is given by the decay mode of the two W bosons, either leptonic , $\mathrm{W} \rightarrow \mathrm{lv}$ or hadronic $\mathrm{W} \rightarrow \mathrm{q} \overline{\mathrm{q}}$.

The electroweak single-Top production, although non-dominant is far from being negligible with a total cross section of about one third of the pair production and constitute an excellent source of naturally polarised Top quarks. Three different mechanisms contribute to the single-Top production: the $\mathrm{W}$-gluon fusion process including the $\mathrm{t}$ channel contribution $(\sigma(\mathrm{Wg})=240 \mathrm{pb}[9][10])$, the associated production of a Top quark and a W $(\sigma(\mathrm{Wt})=60 \mathrm{pb}[9][11])$ and the $\mathrm{s}$ channel process $\left(\sigma\left(\mathrm{W}^{*}\right)=10 \mathrm{pb}[9][10]\right)$. In proton-proton collisions, these single Top cross-sections are not charge symmetric due to the initial parton distribution which can be exploited in the LHC analyses.

\subsection{Motivations for Top physics}

The motivations for Top physics at LHC and at Tevatron are the same. What is the true nature of the Top quark? Does it behave as expected from the Standard model? Is there some particular features in the Top quark production or/and decay indicating new physics beyond the Standard model?

ATLAS and CMS will take benefit from higher performance detectors and much larger Top sample leading to measurements whose precision will be rapidly limited by systematic uncertainties. The advantage of CDF and D0 after years of data taking is the deeper understanding of the detector allowing to reduce significantly the systematics in the measurements. These experiments examined several properties of the Top quark and demonstrated that hadrons colliders are able to perform precision measurements with large Top samples.

A detailed review on Top quark physics potential at the LHC can be found in Ref. [12]. One of the first important measurements is an accurate determination of the Top mass, through the partial or complete reconstruction of the decay final state. The measurement of the $\bar{t}$ crosssection could provide also another, independent, determination of the Top mass. The Top mass is a fundamental parameter of the standard model. Together with a small set of other parameters, the Top mass is used as an input parameter for the theoretical predictions of the electroweak precision observables. Even if the Top quark mass is known with a better precision than the other quark masses, the current precision achieved on the Top mass measurement is the 
dominant effect on the theoretical uncertainties of the electroweak precision observables. A better experimental precision, of the order of $1 \mathrm{GeV}$ would be very important to scrutinize the Standard model, to derive indirect constraint on the unknown model parameters and to constraint other models like the MSSM model [13]. As an illustration Figure 3 shows the current experimental measurement on the $\mathrm{W}$ and Top masses compared with theoretical predictions for the SM and MSSM.

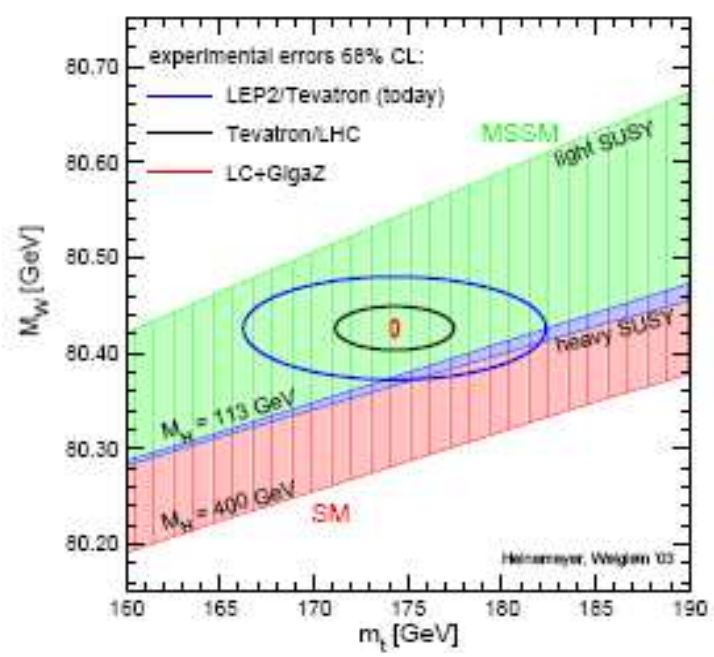

Figure 3 : The current experimental measurement on the $\mathrm{W}$ and Top masses and the expectations in future colliders, compared with theoretical predictions for the SM (red and blue bands) and MSSM (green and blue bands) [13]

Since its mass is much larger than the other quarks, the Top quark plays a privileged role in the electroweak symmetry breaking (EWSB). Any new physics in connection with EWSB would be preferentially coupled to the Top, leading to deviations from the SM in the $\bar{t}$ production rate and distortions in the Top quark kinematics distributions. For example, new resonances or gauge bosons strongly coupled to the Top are expected in a large variety of models, in particular in strong EWSB [14]. These new particles could be revealed in the $\bar{t}$ invariant mass distribution.

The determination of the properties of the electroweak production of the Top will be only possible at LHC, with a separate measurement of the three processes contributing to the single Top production. This will allow a precise direct measurement of CKM matrix element $\mathrm{V}_{\mathrm{tb}}$ and will probe new physics inducing non Standard model weak interactions [15].

Furthermore, the Top signal being one of the most important sources of background to other new physics signal, a detailed understanding of the Top production rates and decay properties will be a necessary path to new discoveries. Since the Top quark decays without forming hadrons, Top quark spin properties are accessible allowing stringent tests on the production and decay mechanisms. This is accessible via the measurements of the $\mathrm{W}$ and Top polarisations and the determination of the size of the Top-antiTop correlation in the case of Top pair production [16]. Here again, any departure from the Standard model would indicate new 
physics at the Top production or/and decay level. Moreover, possibly large flavour changing neutral currents (FCNC) decays of the Top quark such as $\mathrm{t} \rightarrow \mathrm{Zq} ; \mathrm{t} \rightarrow \gamma \mathrm{q}$ and $\mathrm{t} \rightarrow \mathrm{gq}$ could be investigated to search for new physics.

\subsection{Early Top studies}

The first data will be used for commissioning and calibrate the detector in situ with physics processes (see Table 1). The Top production is an ideal laboratory for initial studies. In addition to an abundant production rate, the 'lepton+jets' channel is easy to trigger, and high purity samples of Top events can be extracted with simple selection criteria. This channel is of particular interest since it contains two b-jets, two light jets, one lepton and one neutrino (Figure 4). It deals with many detector characteristics and performances related to lepton momentum and identification, jet reconstruction and calibration, missing energy and b-tagging. So, Top events will be very useful to give feedback on detector performances and as a calibration tool. In addition, precision measurement in the Top sector will be mainly driven by the performances achieved on the jet energy scale and b-tagging. It is of great importance to understand these key points using all relevant physics processes including Top events before performing initial measurements as the Top mass determination.

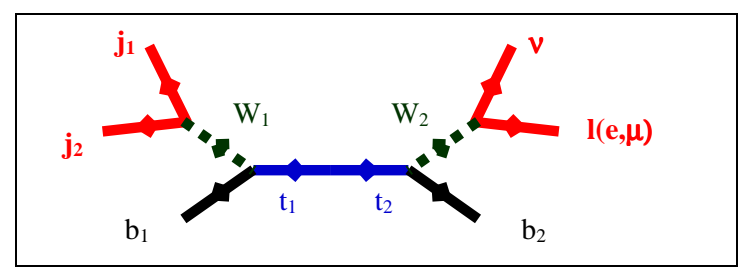

Figure 4 : schematic diagram for the Top decay in the 'lepton+jets' channel

The golden plated channel $\quad \overline{\mathrm{t}} \rightarrow \mathrm{WbWb} \rightarrow \mathrm{lvbbjj}$ (Figure 4) contribute to about $30 \%$ ot the Top decay channel when considering only muons and electrons in the final state. Even at very low luminosity $\left(10^{32} \mathrm{~cm}^{-2} \mathrm{~s}^{-1}\right)$, more than 50 'lepton+jets' events per day after trigger and selection could be obtained to perform studies. The observation of the Top signal should be very fast. The trigger for this channel is based on the search of an isolated lepton. As an example, the main associated high level trigger menus in ATLAS request a muon with $\mathrm{P}_{\mathrm{T}}>20 \mathrm{GeV}$ or an electron with $\mathrm{P}_{\mathrm{T}}>25 \mathrm{GeV}$ [17]. A typical selection requires an isolated lepton, a missing energy above $20 \mathrm{GeV}$, and four jets with a $\mathrm{P}_{\mathrm{T}}$ greater than $40 \mathrm{GeV}$. The physical background is reduced to a negligible level by asking for the presence of one or two b-jets among the four jets. The $\mathrm{W}$ and Top reconstruction on the hadronic side is very simple, through the combination of two light jets (W) or two light jets + one b-tagged jet (Top). The main contributing background comes from wrong combinations of jets in the Top signal.

Even in a pessimistic case where no b-tag could be applied, it has been shown [18] that a clean sample of several thousand events will be available in few weeks during the first data taking (Figure 5). 


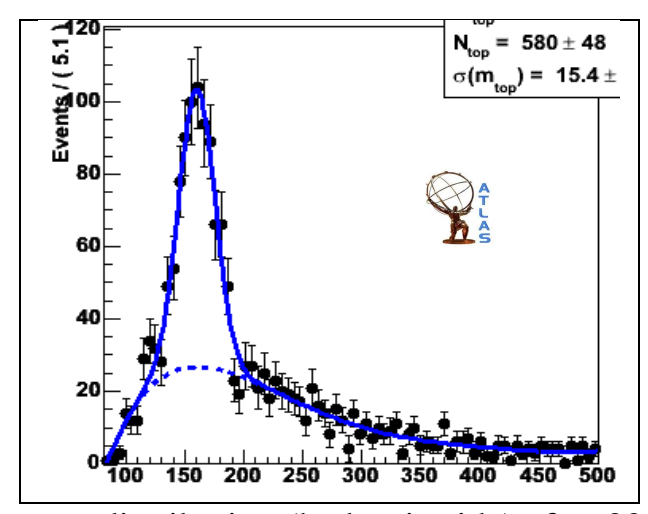

Figure $5 \mathrm{M}\left(\mathrm{jjb}\right.$ ) invariant mass distribution (hadronic side) after $300 \mathrm{pb}^{-1}$ of collected data and with a selection of "lepton+jets" events without applying b-tagging.

The Top signal can be exploited to get the light jet energy scale. A high purity sample (up to $80 \%$ purity) of $\mathrm{W} \rightarrow \mathrm{jj}$ decays originating from the hadronic decay of the Top can be extracted [19]. The initial performance on the jet energy scale is expected to be of the order of $10 \%$ (see Table 1). A light-jet systematic bias in the light jet absolute scale induces a shift on the observed $\mathrm{W}$ mass peak. This allows a determination of the correction factors $\alpha(E)=\frac{E_{\text {parton }}}{E}=f(E)$ needed to properly recalibrate the light-jet scale. To first approximation, the bias on the energy response from the calorimeters will depend on the jet energy and on the direction ( $\eta$ essentially). Only the energy dependence has been studied so far. The overall $\mathrm{W}$ sample is splitted in different $\mathrm{W}$ samples according to the jet energy of the associated jets. The absolute jet scale in function of the energy is then determined by extracting the peak value of the reconstructed $\mathrm{W}$ mass. This procedure does not require any hypothesis on the scale function and can be used as a general calibration function valid even outside Top physics analyses involving light jets. The precision obtained with a sample corresponding to $300 \mathrm{pb}^{-1}$ is about 2-3 $\%$ with a potential precision of the order of the per cent with more data.

A powerful b-tagging needs a precise alignment of the tracking elements. The statistical precision will be reached after few days of operation, but it will take a long time to understand all systematics (about one year to reach the nominal precision on the alignment). Since a Top signal can be extracted without b-tagging, it is possible to perform studies on the b-tagging properties. A tight selection in the $\mathrm{W}$ and Top mass distributions enhances the probability to choose the appropriate jets belonging to the hadronic decay of the Top. The other Top in the event with a leptonic $\mathrm{W}$ decay has an additional jet which has not yet been taken into account in the reconstruction and which must be a b-jet if correct assignments have been made previously. In such a way, a clean sample of b-jets is selected from the Top signal and can be used to btagging studies. 


\subsection{Early Top Physics goals at the LHC}

A detailed review on Top quark physics potential at the LHC can be found in Ref. [12] [20] and soon in [21]. In addition, dedicated aspects to Top production [22][23], Top mass measurements [24][25], Top properties [26], single-Top studies [27] and LHC sensitivity to Top properties beyond the Standard model [28] could also be found in these proceedings.

A rich Top physics program will be open as soon as first steps up to the commissioning phase with first data is successfully passed. The amount of data selected by the ATLAS and CMS experiment with an integrated luminosity of $10 \mathrm{fb}^{-1}$ is quite substantial (see Table 3 ).

\begin{tabular}{|l|c|}
\hline \multicolumn{1}{|c|}{ Channel } & $\begin{array}{c}\text { Selected events for } \\
10 \mathrm{fb}^{-1}\end{array}$ \\
\hline$\overline{\mathrm{t}} \rightarrow \mathrm{WbWb} \rightarrow$ lvbbjj & $70 \mathrm{~K}$ \\
\hline $\mathrm{t} \overline{\mathrm{t}} \rightarrow \mathrm{WbWb} \rightarrow$ lvbbjj high $\mathrm{P}_{\mathrm{T}}$ sample & $3,6 \mathrm{~K}$ \\
\hline$\overline{\mathrm{t}} \rightarrow \mathrm{WbWb} \rightarrow$ lvblvb & $20 \mathrm{~K}$ \\
\hline $\mathrm{t} \overline{\mathrm{t}} \rightarrow \mathrm{WbWb} \rightarrow$ jjbbjj high $\mathrm{P}_{\mathrm{T}}$ sample & $3,4 \mathrm{~K}$ \\
\hline Single top t channel & $2,5 \mathrm{~K}$ \\
\hline Single top Wt channel & $1,5 \mathrm{~K}$ \\
\hline Single top s channel & $0,5 \mathrm{~K}$ \\
\hline
\end{tabular}

Table 3: examples of the amount of Top selected event per experiment $\left(10 \mathrm{fb}^{-1}\right)$ in various channels.

Most of the measurements will have a negligible statistical error and the main systematic limitations will originate from the jet scale (light an $b$ jets), final states radiation (FSR) and from the luminosity measurement uncertainty. First LHC goals with $10 \mathrm{fb}^{-1}$ are to perform:

- A Top mass measurement with a precision of the order or below $1 \mathrm{GeV}$.

- A tt production cross section measurement with a precision below $10 \%$.

- Tests of the Top production and decay mechanisms with W polarisations (Top spin correlation) at the level of $1-2 \%(3-5 \%)$.

- Studies on the $\overline{\mathrm{t} t}$ invariant mass.

- Search for FCNC decays, improving current results by a factor 100.

- A measurement of the cross sections of the three single Top production mechanisms, and a direct measurement of the CKM matrix element $\mathrm{V}_{\mathrm{tb}}$.

\section{Conclusions}

LHC is on the road. The LHC machine, the ATLAS and CMS detectors are close to the final installation phase, first collisions being scheduled in Summer 2007. The initial measurements are expected in two years from now, but a huge work is needed to understand the 
detectors and to control the systematics ( $\mathrm{p}$-p collisions, PDF, and detector related) before to get the first results. Early Top signal will be also critical for commissioning the detectors.

LHC has a great potential for Top physics. An enormous amount of Top quarks will be produced at LHC: 10 days at $10^{33} \mathrm{~cm}^{-2} \mathrm{~s}^{-1}$ is equivalent to 10 years at the Tevatron for Standard model processes. As a consequence measurements will be made with a negligible statistical uncertainty in most of the cases. Some of the early LHC physics results, and early sensitivity to new physics could come from Top physics. With the increase in luminosity up to $10^{34} \mathrm{~cm}^{-2} \mathrm{~s}^{-1}$, LHC will offer a unique opportunity to improve our knowledge on the Top quark and to open a window on physics beyond the Standard model.

\section{References}

[1] http://lhc.web.cern.ch/lhc/

[2] ATLAS Technical proposal CERN/LHCC/94-43

[3] CMS Technical proposal CERN/LHCC/94-38

[4] F. Gianotti and M. Mangano, LHC physics: the first one-two years, CERN-TH-PH/2005-072, [hep-ph/0504221]

[5] http://aenews.cern.ch/

[6] http://cmsinfo.cern.ch/Welcome.html/Timeline/Archive/Archive2006/PicturesOfTheWeek/Pictu resOfTheWeek2006.html

[7] G. Dissertori, LHC expectations (Machine, Detectors and Physics), [hep-ex/0512007]

[8] N. Kidonakis and R. Vogt, Phys Rev D68 (2003) 114014; Eur. Phys. J. C33 (2004) s466

[9] Z. Sullivan, [hep-ph/0408049]

[10] J. Campbell, K Ellis and F Tramontano, Single-top production and decay at NLO, [hep$\mathrm{ph} / 0408158]$

[11] J. Campbell, F Tramontano, NLO corrections to Wt production and deca, [hep-ph/0506289]

[12] G. Altarelli, M.L. Mangano, Workshop on Standard Model Physics (and more) at the LHC, CERN-2000-004(2000)

[13] S Heinemeyer and G Weiglein, The MSSM in the light of precision data, [hep-ph / 0307177]

[14] C T. Hill and E. H. Simmons, Strong Dynamics and Electroweak Symmetry Breaking, [hep$\mathrm{ph} / 0203079]$

[15] T.Tait, C.-P.Yuan, Phys.Rev. D63 (2001) 0140018

[16] F. Hubaut et al, ATLAS sensitivity to top quark and W bosonpolarisation in ttbar events, , [SNATLAS-2005-052] and references therein

[17] ATLAS coll., High level trigger Data acquisition and controls, CERN/LHCC/2003-022

[18] S. Bentvelsen and M. Cobal, Top studies for the Atlas detector Commissioning, ATL-PUB-2005024

[19] I. Borjanovic et al., Investigation of the Top mass measurements with the ATLAS detector at LHC, SN ATLAS-2004-040

[20] ATLAS coll., ATLAS Detector and Physics Performance Technical design report, CERNLHCC-99-14-15

[21] CMS coll., Physics TDR in preparation, to be published in 2006

[22] B. Kersevan, ttbar Production at ATLAS and ttbar Monte Carlos, these proceedings

[23] R Chierici, ttbar Production at CMS, these proceedings

[24] A.I. Etienvre, Top mass measurement at the LHC, these proceedings

[25] J Heyninck, A Kinematic Fit in CMS \& the use of Top Quarks for Calibration, these proceedings

[26] B. Resende, Top properties within the SM, these proceedings

[27] A Giammanco, single-Top production at the LHC, these proceedings

[28] N.Castro, Top properties beyond the SM, these proceedings 